\title{
Anesthésie-réanimation en chirurgie thoracique
}

\author{
Jean Bussières, Marc Leone. Collection Verte. Arnette, 2017; 464 pages. \\ ISBN : 978-2-7184-1383-9
}

\author{
Andrew Owen, MD
}

Received: 21 September 2017 / Accepted: 11 October 2017/Published online: 2 November 2017

(c) Canadian Anesthesiologists' Society 2017

Avec l'utilisation de nouvelles technologies et l'évolution concomitante des techniques en chirurgie thoracique, les lignes directrices de l'anesthésie pour ce type de chirurgie ont bien évolué. Ainsi, il existe un réel besoin de textes décrivant ces nouvelles pratiques, car l'anesthésie thoracique n'est plus 'le petit frère' de l'anesthésie cardiaque et ne se satisfait plus de quelques chapitres à la fin d'un traité sur cette dernière.

En effet, depuis une trentaine d'années déjà, il existe d'excellents ouvrages en anglais abordant le sujet d'une façon exhaustive. Par contre, à ma connaissance, le traité de Bussières et Leone représente le seul ouvrage d'anesthésie-réanimation en chirurgie thoracique rédigé dans la langue de Molière. Leur travail est le résultat d'un effort multidisciplinaire, mettant en avant une collaboration entre anesthésistes, chirurgiens, pneumologues et radiologues issus de la francophonie européenne et nord-américaine. Ainsi, cet ouvrage offre l'avantage de perspectives diverses sur les sujets présentés.

Visant un lectorat moins vaste que les publications anglaises, le livre se positionne nécessairement comme 'hybride' - ni livre de poche, ni texte de référence exhaustif, mais plutôt un compromis entre ces deux pôles.

Cet ouvrage parvient à relever le défi, dans l'ensemble des sujets abordés au fil de ses 37 chapitres de synthèse (entre 4 et 14 pages chacun), d'allier concision et exhaustivité - un exploit pour le moins impressionnant. Ces chapitres incluent notamment de bonnes descriptions de procédures thoraciques spécifiques (résection trachéale, fistule bronchopleurale, etc.). Par contre, le chapitre traitant

A. Owen, MD ( $\square)$

McGill University Health Centre, Montréal, QC, Canada

e-mail: andrewowenmd@hotmail.com de la physiologie pulmonaire, bien que rédigé de manière très compréhensible, souffre peut-être d'un excès de concision.

Certains chapitres (notamment ceux portant sur l'isolation pulmonaire, la bronchoscopie interventionnelle, et le lavage pulmonaire), ont une excellente mise en page accompagnée de belles illustrations et sont très agréables à lire. En revanche, plusieurs autres chapitres auraient bénéficié de meilleures illustrations. Par ailleurs, les descriptions de programmes de récupération accélérée ou de stratégies ventilatoires, par exemple, sont moins plaisantes à la lecture car elles comportent un texte très dense où les sous-titres et sections font défaut, ce qui rend donc plus difficile l'identification des points clefs et des informations importantes. Enfin, un tableau décrivant l'algorithme de la gestion de l'hypoxémie pendant la ventilation unipulmonaire aurait ajouté de la valeur à ce chapitre.

Des sujets récents tels que l'anesthésie et le cancer et les effets du type d'anesthésie sont présentés mais, en raison du manque de données en chirurgie thoracique, et bien que d'intéressantes questions soient posées, les réponses sont laissées largement en suspens.

Parmi les limites de cet ouvrage, citons l'absence de version électronique, de matériel vidéo supplémentaire et, incroyablement, l'absence d'un index. La description écrite de la pose d'un drain thoracique est certes utile mais, si une image vaut mille mots, une vidéo en vaut dix mille. De plus, la couverture souple du livre s'abime rapidement après quelques voyages seulement dans un sac à dos.

Pour l'anesthésiste qui prend en charge de manière occasionnelle des patients subissant une chirurgie thoracique ou pour le résident (interne) en formation qui cherche un livre en français, cet ouvrage constitue 
indubitablement un bel outil. On trouve dans ses pages un excellent point de départ, à partir duquel, au besoin, le clinicien pourra approfondir ses connaissances en se référant à d'autres sources.

Conflit d'intérêt Aucun.
Responsabilité éditoriale Cet article a été traité par Dr Étienne de Médicis, rédacteur de langue française, Journal canadien d'anesthésie. 